\title{
Factorial study on the future freeze-thaw risk in solid masonry walls in Brussels subjected to RCP8.5.
}

\author{
Isabeau Vandemeulebroucke $^{\mathrm{a} *}$, Steven Caluwaerts ${ }^{\mathrm{b}, \mathrm{c}}$, Nathan Van Den Bossche ${ }^{\mathrm{a}}$ \\ a Building Physics Group, Faculty of Engineering and Architecture, Ghent University, Ghent, Belgium \\ b Atmospheric Physics Group, Faculty of Sciences, Ghent Univerisity, Ghent, Belgium \\ c Department Meteorological and Climatological Research, Royal Meteorological Institute of Belgium, Uccle, Belgium
}

\begin{abstract}
The existing building stock is responsible for $40 \%$ of the European energy use. One measure of improving old building's energy efficiency is the application of thermal insulation, often at the interior façade surface. This alters the hygrothermal behaviour of the masonry and changes the risk for freeze-thaw damage. Besides, the durability of brick masonry is potentially affected by climate change. The latter, however, has not been studied widely. This paper presents a sensitivity analysis on the impact of climate change on the freeze-thaw risk in solid masonry. It is found that the freeze-thaw risk is generally decreasing in Brussels based on climate projections by the ALARO-0 RCM (RCP 8.5). Furthermore, the brick type is recognized as the most important variable when assessing the impact of climate change.
\end{abstract}

Peer-review under the responsibility of the organizing committee of the ICMB21.

Keywords: Climate change; sensitivity analysis; interior retrofit; historical brick; freeze-thaw risk.

\section{Introduction}

In Europe, the existing building stock is responsible for $40 \%$ of the total final energy use [1]. Increasing the thermal performance of building envelopes is a measure to improve the building's energy efficiency. However, thermal retrofits, especially interior retrofits, are not always compatible with present wall composition and materials [2]. They alter the wall's hygrothermal behaviour and may entail damage risks, e.g. freeze-thaw action. Many works studied the durability of retrofitted historical buildings, but the results are not univocal according to Campbell et al. [3]. Furthermore, climate change as well may alter the damage risk in historical masonry [4]. Note that this is not widely studied, and HAM simulations based on climate projections remain scarce.

To guarantee the long-term durability of retrofitting solutions, it is necessary to evaluate the change in future damage risk. However, there may be large uncertainties concerning the specifics of the historical masonry wall. Calle [5] found that the wall thickness, brick material properties, rain exposure and orientation have a dominant impact on the durability of uninsulated brick masonry. This study provides a sensitivity analysis on the freeze-thaw risk of solid masonry, before and after interior retrofitting, accounting for climate change.

\section{Methodology}

Climate data: The climate projections originate from the ALARO-0 Regional Climate Model (RCM) with a resolution of $4 \mathrm{~km}$. This study considers the location of Brussels $\left(50.8^{\circ} \mathrm{N} ; 4.3^{\circ} \mathrm{E}\right)(\mathrm{BE})$. The indoor climate is computed based on EN 15026.

Wall assembly: A south-west (SW) orientated historical solid masonry wall with varying thickness is studied without insulation, and with mineral wool insulation at the interior side. The interior finish is either gypsum plaster (uninsulated wall) or gypsum board (insulated wall). The insulated wall is featured with a vapour barrier ( $\mathrm{sd}=2.3 \mathrm{~m})$. The masonry is considered as homogeneous. Sensitivity analysis: The following variables are considered in the sensitivity analysis by means of 1D simulations in Delphin 6.1: 2 time periods, 4 masonry thicknesses, 4 brick materials, 4 insulation thicknesses (including the uninsulated case), and 4 rain exposure coefficients. The periods are 1976-2005 and 2070-2099, respectively the historical period and future RCP 8.5 projection, referring to the high radiative forcing scenario. The masonry thickness varies between $200-500 \mathrm{~mm}$ by $100 \mathrm{~mm}$ intervals. The 4 brick materials are the generic cluster materials of historical brick by Zhao. The thicknesses of interior insulation are $0,50,100$ and $150 \mathrm{~mm}$. The rain exposure coefficients are 0.5, 1.0, 1.5 and 2.0. The total sample size is 512 simulations (full factorial). Damage mechanism: The number of critical freeze-thaw cycles $\left(\mathrm{FTC}_{\text {crit }}\right)$, i.e. freeze-thaw cycles exceeding $25 \%$ moisture saturation, at $5 \mathrm{~mm}$ depth from the exterior is evaluated [4]. This arbitrary selected criterion is considered worst-case scenario.

Table 1 . The yearly average number of critical FTC over the sample per period, and the absolute climate change signal.

\begin{tabular}{|r|c|c|c|}
\hline FTCcrit (yearly average) $[-]$ & Historical period & Future period & Climate change signal (absolute) \\
\hline Range & $0-78$ & $0-33$ & $-46-+1$ \\
\hline Mean (standard deviation) & $33(26)$ & $13(11)$ & $-20(15)$ \\
\hline Interquartile range & 45 & 21 & 28 \\
\hline
\end{tabular}

* Corresponding author. +3292643975 Isabeau.Vandemeulebroucke@UGent.be

(C) The Author(s). This is an open access article distributed under the terms of the Creative Commons Attribution License (CC BY) 4.0 https:// creativecommons.org/licenses/by/4.0/, which permits unrestricted use, distribution and reproduction in any medium, provided the original author and source are credited. DOI: 10.14293/ICMB210062 


\section{ICMB21}
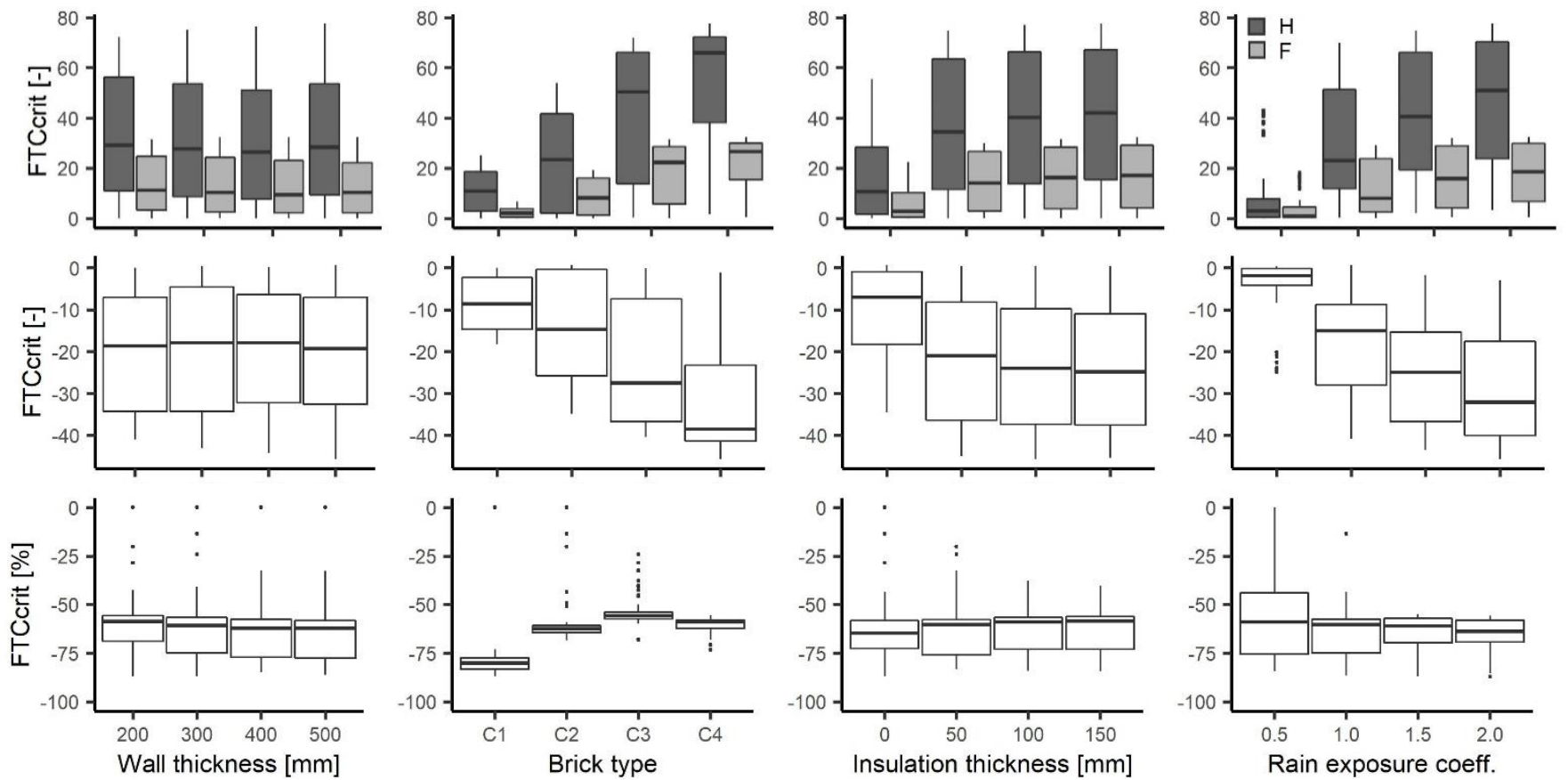

Figure 1. Distribution of yearly average FTCcrit in the historical $(\mathrm{H})$ and future (F) period (top), and the absolute (middle) and relative (bottom) climate change signal. Note that positive changes are excluded from the figures as the maximum change is +1 cycle. (The dots on the figure represent outliers).

\section{Results}

Generally, there is a decrease in freeze-thaw risk in SW-orientated masonry walls in Brussels (Table 1). Next to that, the variability in freeze-thaw risk over the sample per period, and the variability on the absolute change in yearly average FTC $_{\text {crit }}$ is large.

For variations in wall and insulation thickness (except the uninsulated cases), the distribution is quite similar over the sample per period (Fig. 1, top). As the distribution of each variation contains almost the entire sample range for the respective period, the variability in freeze-thaw risk is large. Note that there is a clear difference between the uninsulated and interior retrofitted walls. The brick type and rain exposure coefficient, on the other hand, illustrate different distributions. The freeze-thaw risk becomes generally more important for brick type of cluster 1 up to 4 , and with increasing rain exposure coefficient. This is the case for the historical and future period, though the differences become smaller during the future period. Consequently, the same trends are observed for the absolute climate change signal (Fig. 1, middle).

In contrast, the relative climate change signal is quite similar for the different variations (Figure 1, bottom). The mean change in yearly average $\mathrm{FTC}_{\text {crit }}$ equals ca. $-65 \%$ (standard deviation (sd) 12\%). For the brick type, larger differences in mean changes are observed with values between $-81 \%$ and $-54 \%$, but the variability is smaller except for some outliers, i.e. sd from $4 \%$ to $10 \%$. The rain exposure coefficient of 0.5 displays the largest sd, i.e. $18 \%$, but representing a rather low number of FTC $_{\text {crit. }}$.

\section{Discussion and conclusions}

There is an average decrease by ca. $-65 \%$ in yearly average FTC $_{\text {crit }}$ at $5 \mathrm{~mm}$ depth of solid masonry walls (SW) in Brussels, based on climate projections (RCP 8.5). A large spread is observed for the variation in wall/insulation thickness and rain exposure coefficient. When the brick type is known, the variability is significantly lower. It is found that the brick type is the most important variable when assessing the projected impact of climate change on the freeze-thaw risk in solid masonry.

The authors acknowledge the Royal Meteorological Institute of Belgium for providing the climate projections. Alongside, Isabeau Vandemeulebroucke acknowledges the support of the Research Foundation - Flanders (FWO) (1S90420N).

\section{References}

[1] Buildings Performance Institute Europe. (2011). Europe's buildings under the microscope - A country-by-country review of the energy performance of buildings.

[2] Steskens, P., Vanhellemont, Y., \& Van Den Bossche, N. (2015). A decision making tool for the energy efficient refurbishment of residential buildings. Energy Procedia 78. 997-1002.

[3] Campbell, N., McGrath, T., Nanukuttan, S., \& Brown, S. (2017). Monitoring the hygrothermal and ventilation performance of retrofitted clay brick solid wall houses with internal insulation: Two UK case studies. Case Studies in Construction Materials, 7, 163-179.

[4] Vandemeulebroucke, I., Calle, K., Caluwaerts, S., De Kock, T., \& Van Den Bossche, N. (2019). Does historic construction suffer or benefit from the urban heat island effect in Ghent and global warming across Europe? Canadian Journal of Civil Engineering, 46(11), $1032-1042$.

[5] Calle, K. (2020). Renovation of Historical Facades: The Rescue or the Kiss of Death? [PhD thesis]. Ghent University, Belgium. 